\title{
LA CAMPAGNE FETCH ÉTUDE DES ÉCHANGES OCÉAN-ATMOSPHÈRE DANS LE GOLFE DU LION
}

\author{
Danièle Hauser ${ }^{(1)}$, Hélène Dupuis ${ }^{(2)}$, \\ Xavier Durrieu de Madron ${ }^{(3)}$, Claude Estournel ${ }^{(4)}$, \\ Cyrille Flamant ${ }^{(5)}$, Jacques Pelon ${ }^{(5)}$, Pierre Queffeulou ${ }^{(6)}$ \\ et Jean-Michel Lefèvre ${ }^{(7)}$ \\ (1) Centre d'étude des environnements terrestre et planétaires (CETP), \\ CNRS et université de Versailles - Saint-Quentin-en-Yvelines, \\ 10-12, avenue de l'Europe, 78140 Vélizy \\ (2) Département de géologie et d'océanographie (DGO), \\ CNRS et université de Bordeaux, Talence \\ (3) Centre de formation et de recherche sur l'environnement marin \\ (Cefrem), CNRS et université de Perpignan, Perpignan \\ (4) Laboratoire d'aérologie (LA), CNRS et université Paul-Sabatier, \\ Observatoire Midi-Pyrénées, Toulouse \\ (5) Service d'aéronomie (SA), CNRS et université Pierre-et-Marie-Curie, \\ Paris \\ (6) Ifremer, Département Océanographie spatiale, Brest \\ (7) Météo-France, Service central d'exploitation de la météorologie, \\ Toulouse
}

La campagne Fetch s'est déroulée en mars et en avril 1998 dans le golfe du Lion en Méditerranée. Elle a réuni des chercheurs de la communauté française et internationale. Elle avait pour objectifs d'étudier les échanges entre l'océan et l'atmosphère, la circulation océanique à moyenne échelle et d'améliorer l'apport de la télédétection pour l'estimation du vent, des vagues et des flux à la surface de l'océan. La particularité des conditions du golfe du Lion (vents régionaux intenses comme le mistral et la tramontane, courant liguro-provençal, faible étendue du plateau continental) a été prise en compte pour définir les objectifs détaillés de la campagne et la stratégie de mesure associée. Cet article rappelle les objectifs de l'expérience et présente les moyens mis en auvre ainsi que les premiers résultats obtenus.

ABSTRACT The Fetch experiment; a study of atmosphere-ocean fluxes in the Golfe du Lion

The Fetch experiment took place in March-April 1998 in the Golfe du Lion (Mediterranean sea) and was carried out by French and international scientists. Its objectives were to study the exchanges at the air-sea interface, oceanic circulation on the medium scale and the improvement in the use of remotesensing to estimate wind, waves and fluxes at the sea surface. The special conditions to be found in the experimental area (strong local winds like the Mistral and Tramontane, the presence of the Liguro-Provençal current and small extent of the continental shelf) had to be taken into account when the detailed objectives and the strategy were defined. In this paper we summarize the objectives of the experiment, means used and give preliminary results. 


\section{Fetch et âge des vagues}

Le fetch est relatif à l'effet du vent sur la mer. Il désigne la distance d'action du vent sur la mer et, par extension, sa durée d'action.

Lorsque le fetch est court, les vagues sont en phase de croissance sous l'action du vent et leurs caractéristiques (amplitude, longueur d'onde) dépendent alors à la fois de la vitesse du vent et du fetch. On parle de mer jeune. Inversement, lorsqu'un vent constant a soufflé suffisamment longtemps ou sur une longue distance, leur amplitude et leur longueur d'onde ne dépendent plus que de la vitesse du vent. On parle alors de mer pleinement développée ou de mer vieille.

On caractérise également l'âge des vagues par le rapport entre la vitesse de phase des vagues dominantes et la vitesse du vent. Lorsque ce rapport est petit devant I'unité (vagues jeunes), le bilan d'énergie est dominé par le transfert d'énergie du vent vers les vagues (phase de croissance). Inversement, lorsque ce rapport est proche de l'unité (vagues vieilles), l'énergie transférée par le vent est compensée par la dissipation (déferlement) et l'amplitude des vagues se stabilise.
Les échanges d'énergie à la surface de l'océan constituent des processus majeurs dans le fonctionnement du système océan-atmosphère. Pour la modélisation atmosphérique et océanique ainsi que pour la modélisation couplée du système, ils doivent être représentés le plus précisément possible car ils influent sur la dynamique et la thermodynamique des premières couches de l'atmosphère (couche limite atmosphérique) et de l'océan (couche mélangée). On distingue deux types d'échanges : ceux qui sont dus aux phénomènes turbulents - les flux de quantité de mouvement, de chaleur sensible, de chaleur latente - et ceux qui sont liés aux échanges radiatifs - les flux de rayonnement visible et infrarouge. Ces échanges dépendent des caractéristiques atmosphériques au voisinage de la surface (vent, température, humidité) ou en altitude (couverture nuageuse, etc.), ainsi que des caractéristiques océaniques (température, état de la mer, etc.). Pour progresser dans la connaissance et la mesure de ces échanges, ainsi que pour améliorer leur représentation dans les modèles, la communauté scientifique s'est impliquée depuis plusieurs années dans de grandes campagnes coopératives. En particulier, la communauté française a été à l'initiative de la campagne Semaphore (Eymard et al., 1996). Dans le cadre de l'expérience Fastex en 1997, une composante relative aux flux turbulents a également été mise en place : la campagne Catch (Eymard et al., 1999).

Dans le prolongement de ces campagnes, l'un des objectifs de la campagne Fetch (acronyme construit à partir des mots flux, état de la mer et télédétection en condition de fetch variable) était l'étude des interactions entre l'océan et l'atmosphère et, plus précisément, la quantification et la paramétrisation des flux turbulents de chaleur et de quantité de mouvement à la surface de l'océan. Mais, cette fois-ci, il s'agissait de prendre en compte deux aspects nouveaux : les conditions fréquentes de vent fort et de fetch court liées aux situations de mistral et de tramontane ${ }^{(1)}$, ainsi que l'étude par télédétection des paramètres caractérisant la surface. De façon plus générale, la campagne Fetch, menée en mars et en avril 1998 dans la région du golfe du Lion, par des chercheurs du CNRS, de l'Ifremer, de Météo-France et d'universités françaises et étrangères, visait à répondre à quatre séries d'objectifs complémentaires, tous liés aux échanges à l'interface entre l'océan et l'atmosphère.

Le premier objectif concerne les échanges turbulents et radiatifs à l'interface, la couche limite atmosphérique et les aérosols. À l'heure actuelle, il existe encore d'importantes incertitudes sur la quantification et la représentation des flux turbulents et radiatifs dans les modèles atmosphériques et océaniques. En particulier, on connaît mal la dépendance des flux turbulents par rapport au vent et à l'état de développement des vagues (Geernaert, 1990). C'est pourquoi l'un des objectifs majeurs de Fetch est d'améliorer la mesure et la paramétrisation de ces flux. De façon complémentaire, il s'agit de quantifier les variations spatiales de ces flux en relation avec les caractéristiques de la couche limite atmosphérique et de séparer les différentes causes de ces variations (modification d'écoulement atmosphérique au passage entre la terre et la mer, changement de rugosité dû au développement des vagues, influence de l'échelle synoptique). Enfin, en ce qui concerne les aérosols, il s'agit dans Fetch de mieux quantifier, selon les conditions de vent ou de mer, la nature des aérosols créés localement (extraits de la surface marine) et de ceux qui sont transportés depuis le continent, ainsi que leur part respective.

Le deuxième objectif est relatif aux vagues. D'une part, pour les besoins de la météorologie marine, il est important d'améliorer la prévision de l'état de la mer, dans les conditions de vent fort rencontrées lors des situations de mistral ou de tramontane. D'autre part, les chercheurs veulent mieux comprendre le rôle joué par les vagues dans les échanges turbulents à l'interface. On s'intéresse plus particulièrement aux conditions de développement (fetch limité, par

(1) Le mistral et la tramontane sont tous deux des vents régionaux soufflant depuis la terre vers la mer et liés à la situation météorologique sur l'Europe de l'Ouest. Le mistral, canalisé par la vallée du Rhône, souffle plutôt du nord, tandis que la tramontane souffle plutôt du nord-ouest par la vallée de la Garonne et son prolongement vers la Méditerranée. Il arrive que les deux vents coexistent, conduisant à une convergence au large du golfe du Lion. 


\section{Équipes ayant participé à la campagne Fetch}

\section{France}

Cefrem (CNRS et université de Perpignan)

CETP (CNRS et université de Versailles - Saint-Quentin)

DGO (CNRS et université de Bordeaux 1)

Département Océanographie spatiale (Ifremer)

Division technique de l'Insu

Laboratoire Interactions océanatmosphère (IRPHE et CNRS)

Laboratoire d'aérologie (CNRSuniversité Paul-Sabatier de Toulouse) Laboratoire des sondages électromagnétiques de l'environnement terrestre (CNRS et université de Toulon)

Prévi-Mar (Service central d'exploitation de la météorologie, MétéoFrance)

Centre d'aviation météorologique (CNRM, Météo-France)

Groupe de météorologie expérimentale et instrumentale (CNRM, Météo-France)

Service d'aéronomie (CNRS et université Pierre-et-Marie-Curie de Paris)

\section{Étranger}

Deutsche Zentrum für Luft und Raumfahrt (DLR), Oberpfaffenhofen, Allemagne

Rosenstiel School of Marine and Atmospheric Sciences (Université de Miami, États-Unis)

Finnish Institute of Marine Research, Helsinki, Finlande exemple en cas de mistral) et aux conditions de mer complexe (superposition de houle et de mer du vent), dans lesquelles on connaît mal les caractéristiques des vagues : énergie totale, pic et étalement en fréquence, étalement angulaire. Pour améliorer la prévision numérique de l'état de la mer, on cherche à valider ou à améliorer les paramétrisations actuellement utilisées dans les modèles pour représenter la génération, la croissance et la dissipation des vagues. En Méditerranée, où le relief côtier rend l'écoulement atmosphérique plus complexe, on cherche également à estimer quel peut être l'apport du forçage des modèles de prévision des vagues par des champs de vent à résolution fine obtenus grâce à un modèle régional comme le modèle Aladin de Météo-France. Un autre aspect important concerne l'étude du déferlement et des vagues courtes, dont la longueur d'onde varie de quelques centimètres à plusieurs mètres ; ces processus gouvernent fortement la réponse des instruments de télédétection et jouent un rôle majeur dans le couplage entre le vent et les vagues par rétroaction des vagues sur l'écoulement de l'air.

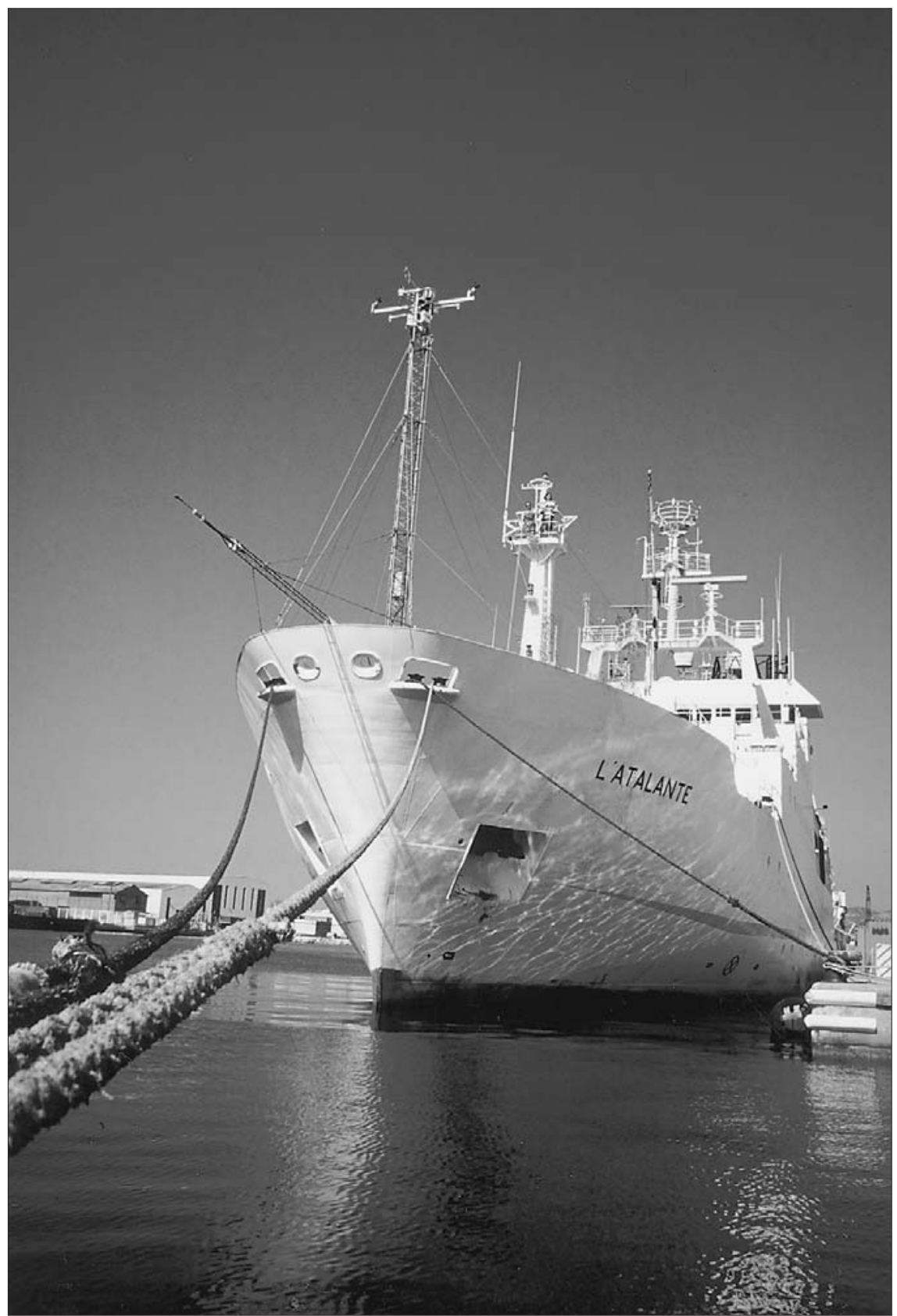

L'Atalante au quai de I'Ifremer, à La Seyne-sur-Mer. À l'avant, le mât instrumenté et la perche permettant une mesure de rayonnement non perturbée par la coque. (Photo G. Lachaud) 
Le troisième objectif se situe dans le domaine des observations par télédétection. Malgré l'essor, durant les dix dernières années, des mesures en télédétection hyperfréquence par les radars et les radiomètres, il reste de nombreuses questions à résoudre. Dans certaines conditions, et notamment près des côtes, plusieurs phénomènes peuvent modifier les relations entre les paramètres géophysiques (le vent et les vagues) et les mesures par télédétection hyperfréquence. C'est notamment le cas pour les modifications de la couche limite atmosphérique au passage terre-mer (variations spatiales de la stabilité atmosphérique et du frottement en surface) et pour l'éventuelle influence de l'état de développement de la mer. Pour mieux quantifier ces processus, l'utilisation de la modélisation physique directe du signal hyperfréquence est un outil performant mais, pour progresser, il faut décrire conjointement les caractéristiques de la surface (spectre des vagues, écume, etc.), les paramètres moyens dans l'atmosphère ou en surface (vent, température, etc.) et les paramètres de télédétection. Par ailleurs, dans le domaine de la télédétection optique, les possibilités prometteuses offertes par les lidars, pour fournir simultanément des paramètres relatifs à la surface et à la couche limite atmosphérique, doivent être confirmées. Enfin, d'une manière générale, l'estimation des flux turbulents à partir de données de télédétection reste balbutiante, même si quelques méthodes ont été proposées récemment.

Enfin, le dernier objectif porte sur la connaissance de la circulation océanique du golfe du Lion et sa modélisation. Il s'agit en particulier de valider et d'améliorer un modèle de circulation qui doit servir à comprendre et à quantifier les mécanismes de transfert d'énergie et de matière dans cette région. Un point important concerne l'étude de la variabilité des courants et l'identification des contributions respectives de trois phénomènes : variations des flux air-mer (notamment en conditions de mistral ou de tramontane), processus de circulation à l'échelle du bassin (courant liguro-provençal) et apports d'eau douce par le Rhône.

DISPOSITIF EXPÉRIMENTAL, DOMAINE D'ÉTUDE ET STRATÉGIE D'OBSERVATION
Figure 1 - Zone de l'expérience Fetch. Les traits gras délimitent approximativement la zone d'intervention maximale des avions et du navire. Le point B représente la position de la bouée Asis de l'université de Miami, pendant toute la campagne, et de la bouée vagues du FIMR, jusqu'au 25 mars. Le point $\mathrm{B}^{\prime}$ indique la position approximative de la bouée FIMR à partir du 25 mars.
Le dispositif de l'expérience comprenait un navire océanographique, une bouée instrumentée et mouillée en un point fixe à environ $60 \mathrm{~km}$ de la côte, deux bouées " houle » et trois avions de recherche. La figure 1 illustre le domaine d'observation. L'utilisation de ces moyens était coordonnée dans le temps et dans l'espace avec les observations des satellites ERS 2 et Topex.

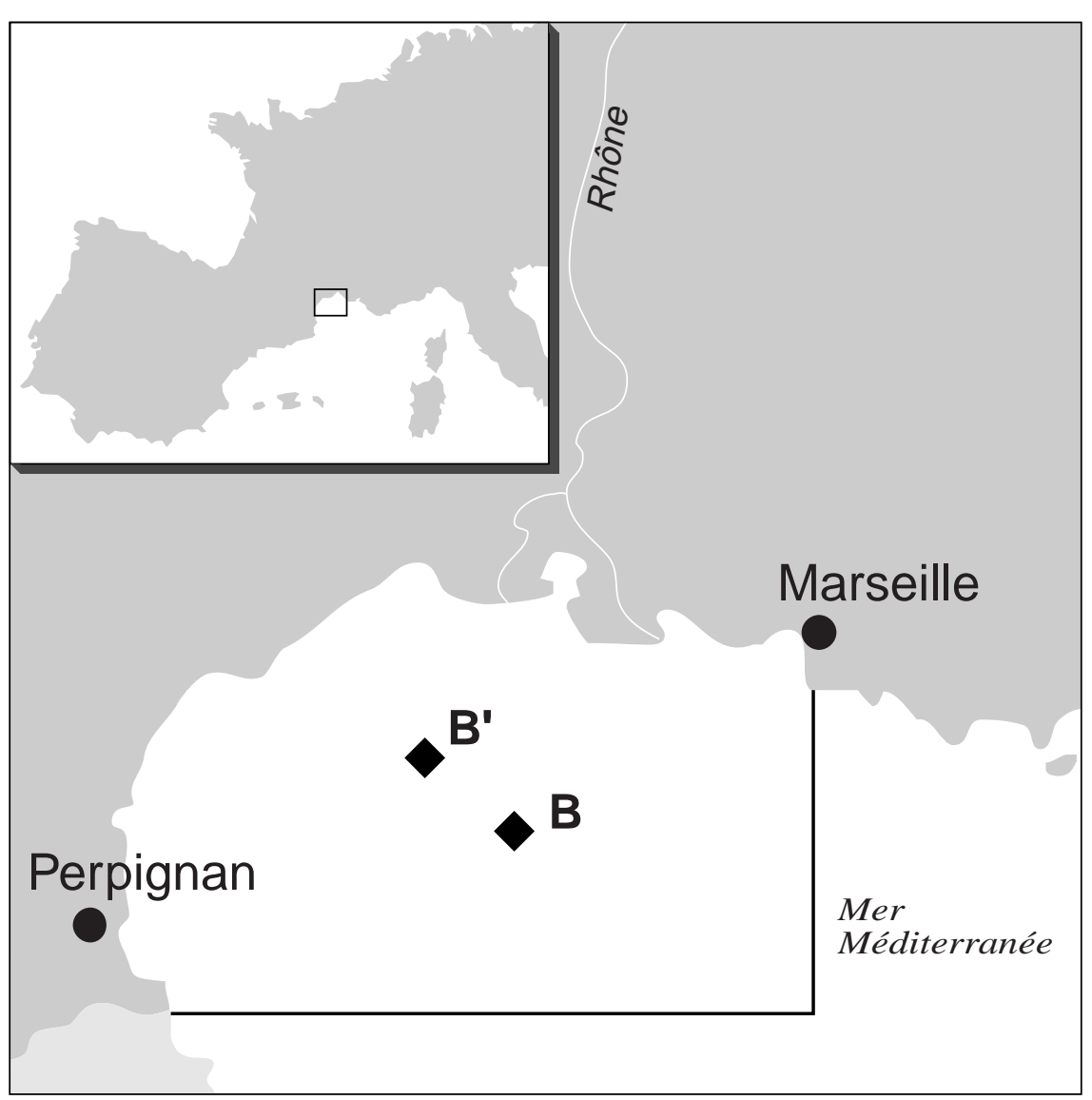




\section{Les observations depuis l'Atalante}

Le navire océanographique français l'Atalante était mis à la disposition de l'expérience par l'Ifremer pour une mission de cinq semaines environ (du 13 mars au 15 avril 1998). Il a été utilisé à la fois pour des mesures dans l'atmosphère, en surface, et pour des mesures d'océanographie physique. Une des particularités du dispositif expérimental a consisté à équiper l'Atalante d'un mât météorologique conçu par le Centre national de recherches météorologiques (CNRM) de Météo-France et instrumenté par Météo-France et le Centre d'étude des environnements terrestre et planétaires (CETP) pour la mesure des paramètres atmosphériques (vent, température, humidité et pression), des flux radiatifs et des flux turbulents de quantité de mouvement et de chaleur.

\section{Les mesures effectuées depuis l'Atalante}

\section{Dans l'atmosphère}

- Pression, vent, température, humidité, rayonnement : capteurs météorologiques sur mât (mesures en continu à environ $18 \mathrm{~m}$ au-dessus de la surface).

- Flux turbulents de quantité de mouvement et chaleur : anémomètre sonique du CNRM et réfractomètre du CETP sur mât (mesures en continu à environ $16 \mathrm{~m}$ au-dessus de la surface.

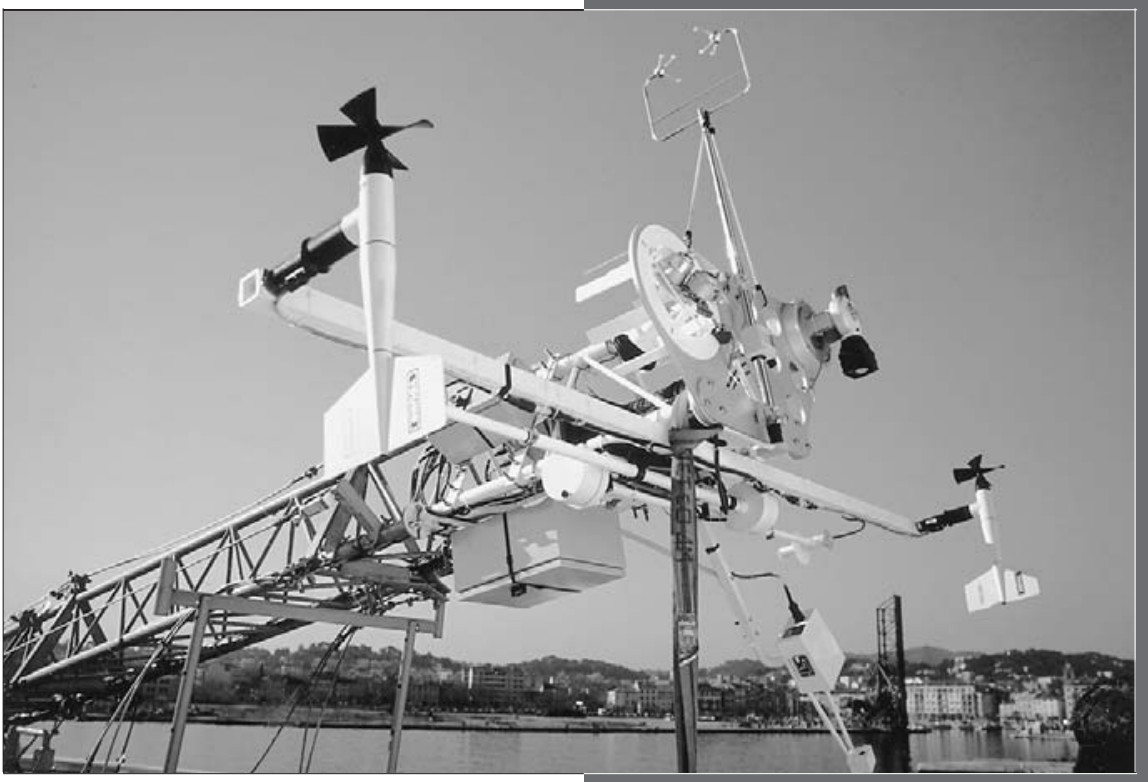

- Profils de vent, température, humidité : radiosondes lancées de façon régulière (deux à quatre fois par jour).

- Aérosols (granulométrie, composition chimique) et gaz DMS (diméthylsulfure) : prélèvements et analyse en laboratoire.

- Contenu en vapeur d'eau et eau liquide de l'atmosphère : radiomètre hyperfréquence Drakkar du CETP, en visée verticale.

En surface

- Température et salinité de surface : thermosalinomètre de laboratoire et instrumentation de base du navire.

- Séréophotographie numérique et images vidéo de la surface.

- Température de brillance en hyperfréquence : observations du radiomètre Drakkar du CETP en visée oblique vers la surface.
La tête de mât et ses différents capteurs avant l'installation du dispositif à la verticale. On distingue deux anémomètres-girouettes et un anémomètre sonique 3D, en haut de l'image (Lachaud, 1998).

\section{Dans l'océan}

- Profils de température, salinité et densité : sondages CTD (Conductivity,

Temperature, Depth).

- Profils de courant : sondage acoustique Doppler.

- Analyse de gaz DMS (diméthylsulfure) dissous dans l'eau.

- Mise à l'eau de courantomètres acoustiques de surface.
La bouée instrumentée Asis

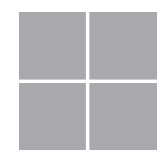

Afin de suivre l'évolution temporelle des conditions atmosphériques et de l'état de la mer en un point fixe, la bouée Asis (Air-Sea Interaction System) décrite par Graber et al. (1999) a été déployée par l'université de Miami pendant toute l'expérience. Elle était mouillée par $90 \mathrm{~m}$ de fond à environ $60 \mathrm{~km}$ des côtes du golfe du Lion (point B sur la figure 1) et était instrumentée pour la mesure de paramètres météorologiques et de surface (vent, température, humidité, pression, température de la surface de la mer), ainsi que pour la mesure du flux de quantité de mouvement et du spectre directionnel des vagues. Cette bouée a été spécialement conçue pour éviter les déplacements par rapport à la surface en cas de vent ou de fortes vagues, afin de limiter les perturbations sur la mesure. De plus, tous les mouvements de la bouée étaient enregistrés à la même cadence d'acquisition que les autres paramètres, ce qui permet de corriger les mesures de façon précise. 


\section{Les bouées « houle »}

Les mesures aéroportées
Deux autres bouées de mesure des vagues, l'une mise à disposition par MétéoFrance et l'autre par le Finnish Institute of Marine Research (FIMR), ont été mises à l'eau dans la zone pour compléter les mesures de vagues à différentes distances de la côte.

Deux avions de recherche de la communauté française (Chalon et al., 1998) étaient également au centre du dispositif expérimental : le Merlin IV de MétéoFrance et le Fokker 27 Arat exploité par l'Institut géographique national (IGN), l'Institut national des sciences de l'univers (Insu) du CNRS, le Centre national d'études spatiales (Cnes) et Météo-France. Les deux avions étaient équipés d'une instrumentation météorologique permettant d'accéder aux caractéristiques moyennes et turbulentes de l'atmosphère.

Chacun des deux avions embarquait aussi de l'instrumentation de télédétection. À bord de l'Arat, le lidar Léandre 2 (Bruneau et al., 1999), développé par l'Insu et le Service d'aéronomie, a été utilisé pour mesurer l'humidité spécifique de l'atmosphère, ainsi que les caractéristiques des vagues courtes générées par le vent. Le Merlin IV embarquait le radar Ressac (Hauser et al., 1992) développé par le CETP pour la mesure des vagues et du vent de surface.

Les vols alternaient des paliers dans l'axe du vent, de la côte jusqu'à une centaine de milles au large, et des sections perpendiculaires à cet axe. Certains passages avaient lieu à basse altitude $(100$ à $300 \mathrm{~m})$ pour étudier la turbulence dans les basses couches.

De façon plus ponctuelle, le Deutsche Zentrum für Luft und Raumfahrt (DLR) a participé à l'expérience avec trois vols du Falcon 20, équipé du lidar Adolar développé pour la mesure du vent dans l'atmosphère.

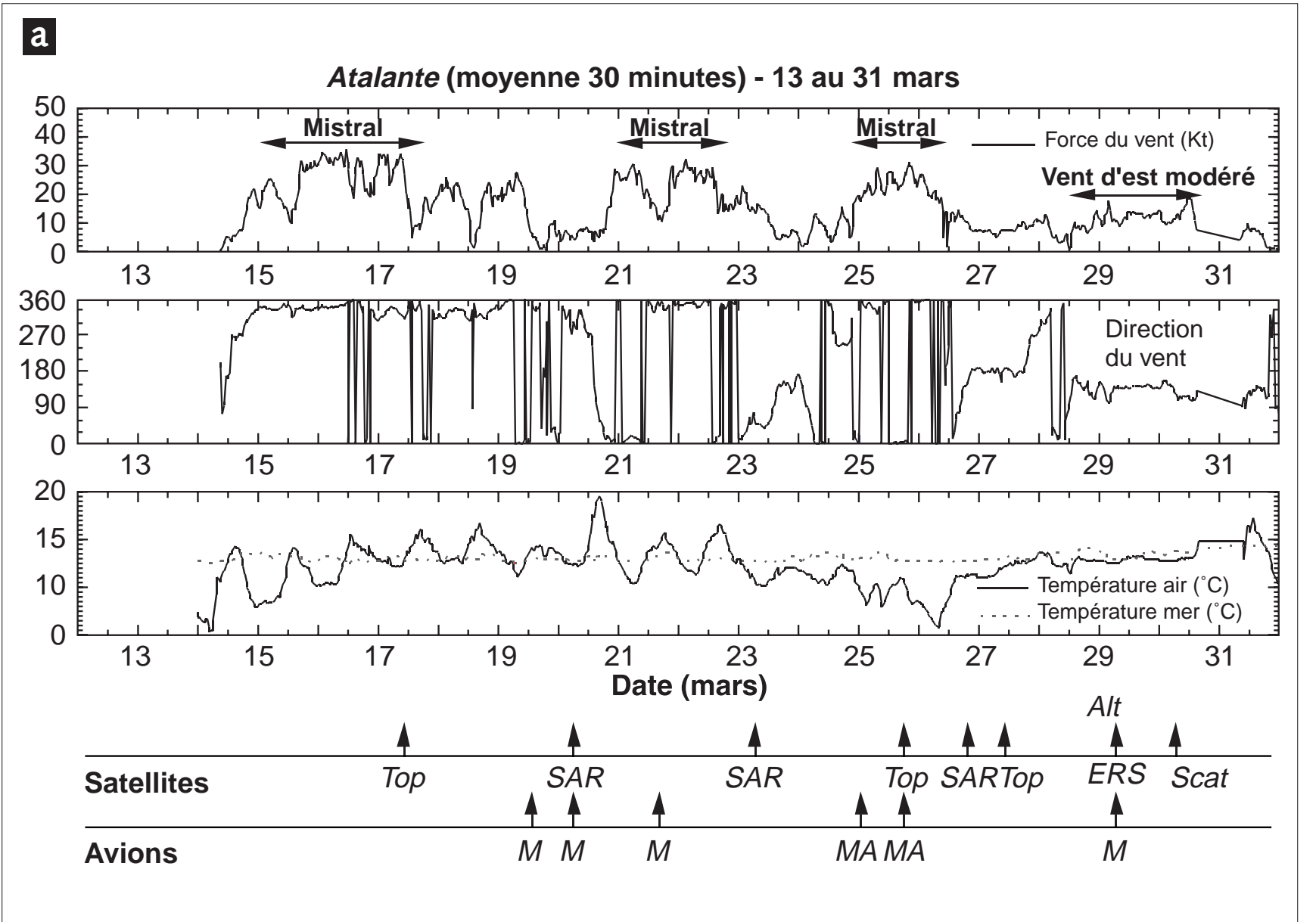

Figure 2 - Séries temporelles des paramètres mesurés sur l'Atalante du 13 au 31 mars (figure 2a) et du 30 mars au 15 avril 1998 (figure 2 b, page suivante). De haut en bas, vitesse du vent en nœuds, direction du vent, température de l'air et de la surface de la mer. En dessous de ces tracés, les flèches indiquent les heures pour lesquelles des données des satellites (mesures par radar) sont disponibles sur la zone Fetch (Top pour Topex, Alt pour altimètre d'ERS 2, SAR pour SAR d'ERS 2, Scat pour diffusiomètre d'ERS 2), ainsi que les heures des vols des avions (M pour Merlin, A pour Arat, F pour Falcon). 
b
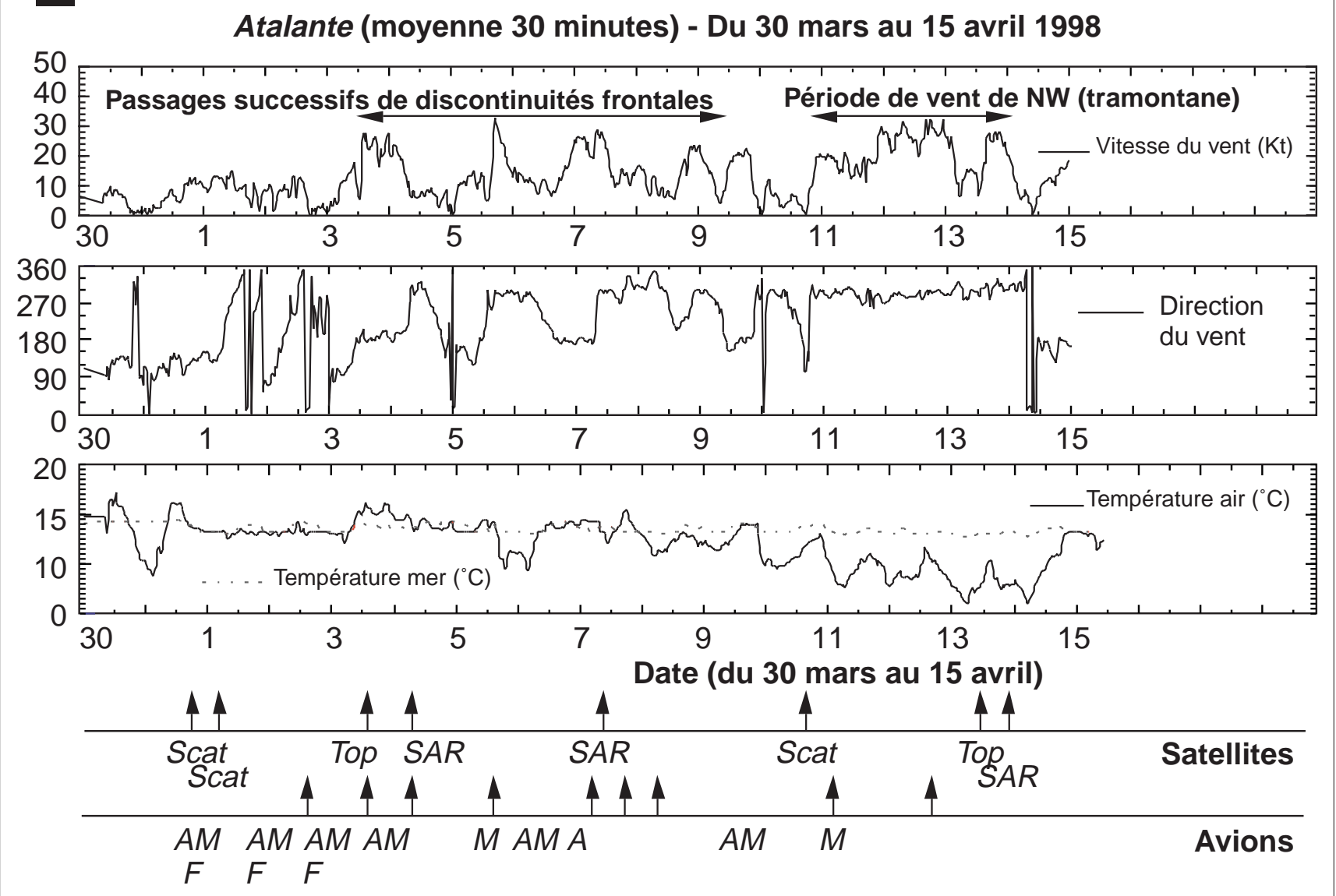

\section{Stratégie d'observation}

La stratégie d'observation a été définie de façon à utiliser les différents moyens d'observation de manière complémentaire. Lors des situations de vent soufflant depuis la côte (fetch limité), le navire a effectué des radiales face au vent sur environ 100 milles, en passant par le point B (figure 1). Le long de ces radiales, il a stationné en plusieurs points pour effectuer des mesures coordonnées dans l'atmosphère, dans l'océan et en surface. Durant ces mêmes situations, les avions étaient mis en alerte pour voler le long de paliers dont l'axe principal était aligné avec la direction du vent. Des passages à haute altitude (2 500 à $6000 \mathrm{~m})$ ont permis de collecter des observations par télédétection (radar, lidar), tandis que des passages à basse altitude $(100$ à $300 \mathrm{~m})$ ont été réalisés pour la mesure des paramètres de la couche limite atmosphérique (paramètres moyens et turbulents).

En dehors de ces épisodes de vent soufflant depuis la côte, l'expérience Fetch a eu pour particularité de coordonner dans le temps et dans l'espace les mesures in situ et les mesures aéroportées avec les observations des satellites ERS 2 et Topex dont les données peuvent servir à déterminer le vent de surface et les caractéristiques des vagues. Chaque fois que l'un de ces satellites survolait la zone, les avions, le navire et les bouées effectuaient des observations concomitantes.

\section{PREMIERS RÉSULTATS} ET BILAN

Nous sommes encore dans la phase d'analyse des données qui, pour la plupart, ont été validées et transmises par les équipes de recherche à une base de données, développée par Medias-France et l'Institut Pierre-Simon Laplace (IPSL). Cette base de données contient également de très nombreuses données opérationnelles archivées pendant la campagne : sorties des modèles de prévision du temps de Météo-France (Aladin et Arpège) et du Centre européen pour les prévisions météorologiques à moyen terme (CEPMMT), sorties des modèles de prévision d'état de la mer de Météo-France (VAG) et du CEPMMT (WAM), images des satellites NOAA et Météosat. 


\section{Analyse hydrologique et courantométrique}

Figure 3 - Champs de courant mesurés à $18 \mathrm{~m}$ sous la surface de la mer le long de la trajectoire de l'Atalante pour deux périodes de la campagne Fetch : en haut, du 13 au 18 mars 1998 ; en bas, du 23 au 29 mars 1998. Analyse effectuée par X. Durrieu de Madron et C. Estournel.
Le bilan des observations (figure 2) montre qu'au début de la campagne, trois événements de mistral ont été documentés, dont deux de façon détaillée. Puis, après une période d'une dizaine de jours de vent faible de sud-est, avec présence de houle certains jours, la fin de la campagne a permis d'observer plusieurs situations de coups de vent de nord-ouest à l'arrière de fronts froids (tramontane temporaire). On dispose ainsi de mesures coordonnées, par bateau, bouées, avions et satellites, dans des situations variées de vent, d'état de la mer et de stabilité atmosphérique. Au total, le navire a effectué 15 radiales dans des situations de mistral ou de tramontane, jusqu'à 120 à $150 \mathrm{~km}$ de la côte, 23 arrêts coordonnés avec des mesures des satellites Topex, ERS et DMSP (équipé du radiomètre hyperfréquence SSMI) et une radiale en situation de vent du large avec houle. De plus, 14 vols des avions Arat et Merlin IV ont été effectués lors de situations de mistral ou de tramontane et 8 dans d'autres conditions, en coordination avec des passages de satellites.

C'est l'analyse combinée de l'ensemble de ces données qui va permettre, dans les deux à trois années à venir, d'atteindre les objectifs scientifiques fixés. S'il est encore trop tôt pour donner des résultats détaillés sur tous les sujets, nous présentons néanmoins ci-après quelques résultats préliminaires.
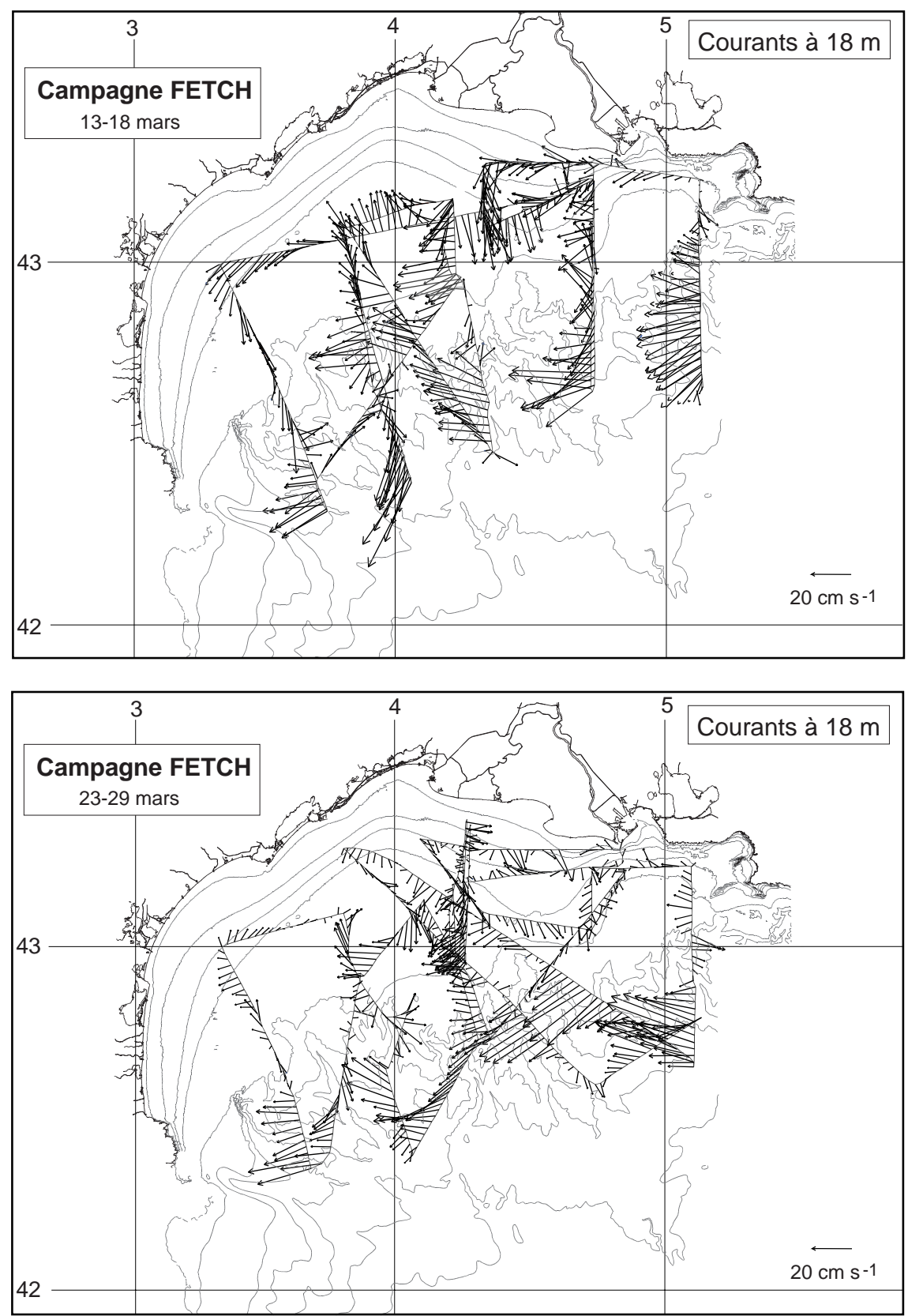
La campagne Fetch a permis d'obtenir 169 profils hydrologiques sur une période d'un mois. Les propriétés des eaux de surface ont également été déterminées en continu au moyen d'un thermosalinomètre dont le traitement était placé sous la responsabilité du Laboratoire de physique et chimie marines (LPCM) de Villefranche-sur-Mer. Ces données montrent en général une colonne d'eau peu stratifiée et un front bien marqué entre les eaux du courant liguro-provençal, au large, et les eaux côtières dont la température, la salinité et la densité sont plus faibles. Les eaux côtières de la partie occidentale du golfe sont cependant plus froides et plus denses, sans doute en raison du vent plus fort dans cette zone. Les mesures de courant obtenues par les profileurs acoustiques à effet Doppler de l'Atalante tout au long de la campagne ont été traitées par le Centre de formation et de recherche sur l'environnement marin (Cefrem). La figure 3 présente une synthèse des courants de subsurface pour deux périodes pendant lesquelles l'ensemble du golfe du Lion a été étudié. Les vitesses de courant tracées sur la figure 3 ont été estimées à partir des moyennes des données brutes sur des distances de $2 \mathrm{~km}$. Si la zone située au-dessus du talus continental est toujours caractérisée par des courants dirigés vers l'ouest, typiques du courant liguro-provençal, il n'en est pas de même des courants sur le plateau continental qui présentent une variabilité spatio-temporelle plus forte le long des trajets effectués. La structure verticale des courants sur le plateau ne fait cependant pas apparaître de rotation importante du courant avec la profondeur. Un profileur de courant qui était mouillé au point $\mathrm{B}$, à 20 mètres au-dessus du fond, a enregistré, pendant l'ensemble de la campagne, le courant dans la couche de fond. Ces données devraient permettre d'étudier l'éventuelle corrélation avec le forçage atmosphérique et de distinguer les variations spatiales et temporelles des courants mesurés depuis le navire.

Le modèle tridimensionnel de circulation océanique côtière Symphonie du Laboratoire d'aérologie est utilisé pour étudier les processus physiques mis en jeu durant la campagne Fetch. L'une des originalités de l'approche consiste à initialiser le modèle par une méthode inverse à partir du champ de densité mesuré. Le forçage par les flux atmosphériques sur l'ensemble du golfe est déduit du modèle à maille fine Aladin de Météo-France, dont les sorties ont été confrontées aux mesures réalisées pendant la campagne. Dans un proche avenir, les caractéristiques des vagues seront également utilisées pour le calcul de la turbulence en surface et dans la couche limite de fond. Grâce au jeu de données recueillies pendant Fetch, nous espérons comprendre l'interaction entre les différents processus et hiérarchiser leur contribution à la circulation côtière dans les différentes conditions météorologiques rencontrées pendant la campagne : remontées d'eau froide à la côte et éventuellement mouvements convectifs générés par les situations de vents forts, mistral et tramontane, soufflant sur une mer plus chaude ; interaction avec la circulation à l'échelle du bassin (courant liguro-provençal et ses prolongements vers le plateau continental) ; panache d'eau douce du Rhône tendant à alléger les eaux du plateau. On cherche également à valider le modèle de turbulence. La couche limite de fond, souvent caractérisée par des eaux plus denses et plus turbides, fait l'objet d'une attention particulière, car elle constitue une voie privilégiée de transfert de la matière particulaire sur le plateau continental.

Estimation des flux de quantité de mouvement et de chaleur

Pendant Fetch, les mesures ont été réalisées à des altitudes différentes sur la bouée ancrée Asis, sur l'Atalante et sur les avions instrumentés, l'Arat et le Merlin IV. L'utilisation complémentaire de ces observations pour des analyses spatio-temporelles est un des points originaux de l'expérience Fetch, mais elle nécessite des études comparatives préalables, visant à valider les différentes estimations de flux. L'analyse conjointe des données de la bouée Asis et de l'Atalante par méthode inertio-dissipative et par méthode de corrélation (voir encadré page suivante) est en cours. Elle doit permettre de mieux quantifier les incertitudes et les erreurs associées à chacune des méthodes. On sait en particulier que la méthode de corrélation est plus sensible aux effets de distorsion d'écoulement d'air autour du navire que la méthode inertio-dissipative, tandis que cette dernière présente des incertitudes plus importantes dans les conditions de vent faible.

Les premiers résultats obtenus à partir des données de l'Atalante et de la méthode inertio-dissipative sont présentés sur la figure 4 où sont tracés les coefficients d'échange turbulent pour chacun des flux, en fonction du vent moyen. 
Mesure des flux turbulents à l'interface air-mer

Les flux de quantité de mouvement, de chaleur et d'évaporation peuvent être estimés à l'interface air-mer à partir d'instruments qui mesurent les fluctuations turbulentes des composantes du vent, de l'humidité et de la température. Ces instruments doivent fonctionner avec une cadence d'acquisition élevée ( $50 \mathrm{~Hz}$ typiquement). Pour les utiliser en milieu marin, ils ne doivent pas être perturbés par les dépôts de sel, les embruns, etc.

Les capteurs employés pour la mesure des fluctuations turbulentes de vitesse du vent sont relativement classiques et identiques à ceux utilisés sur le continent : il s'agit d'anémomètres soniques à trois axes à partir desquels on mesure le temps aller-retour entre trois émetteurs-récepteurs ultrasoniques. En revanche, les instruments classiques qui servent pour les mesures de température et d'humidité de l'air (thermistances, thermocouples, capacitances, psychromètres, etc.) sont difficilement utilisables pour les mesures en mer, en raison des contaminations par les embruns. En France, une méthodologie et une instrumentation originales ont été développées, associant un anémomètre sonique (mesure des composantes du vent et mesure de température liée à la vitesse du son dans l'air) et un réfractomètre hyperfréquence dont la mesure d'indice de réfraction de l'air dépend au premier ordre, au-dessus l'océan, de l'humidité de l'air.

Pour estimer les flux turbulents de quantité de mouvement et de chaleur (latente et sensible) à partir de ces mesures, deux méthodes sont possibles. Si les mesures sont associées à des capteurs permettant I'acquisition des six degrés de liberté du mouvement de la plate-forme (centrale à inertie, gyroscopes, accéléromètres), une estimation des flux turbulents par méthode directe (dite méthode de corrélation) est possible après correction des mouvements de la plate-forme. La deuxième méthode couramment employée audessus la mer est la méthode dite inertio-dissipative. Elle combine la mesure des fluctuations à très haute fréquence (dans la zone inertielle) et l'utilisation de l'équation du bilan d'énergie cinétique turbulente pour estimer les flux turbulents. On s'affranchit dans ce cas des mouvements de la plate-forme en analysant les signaux dans une gamme de fréquence qui est peu perturbée par ces mouvements.
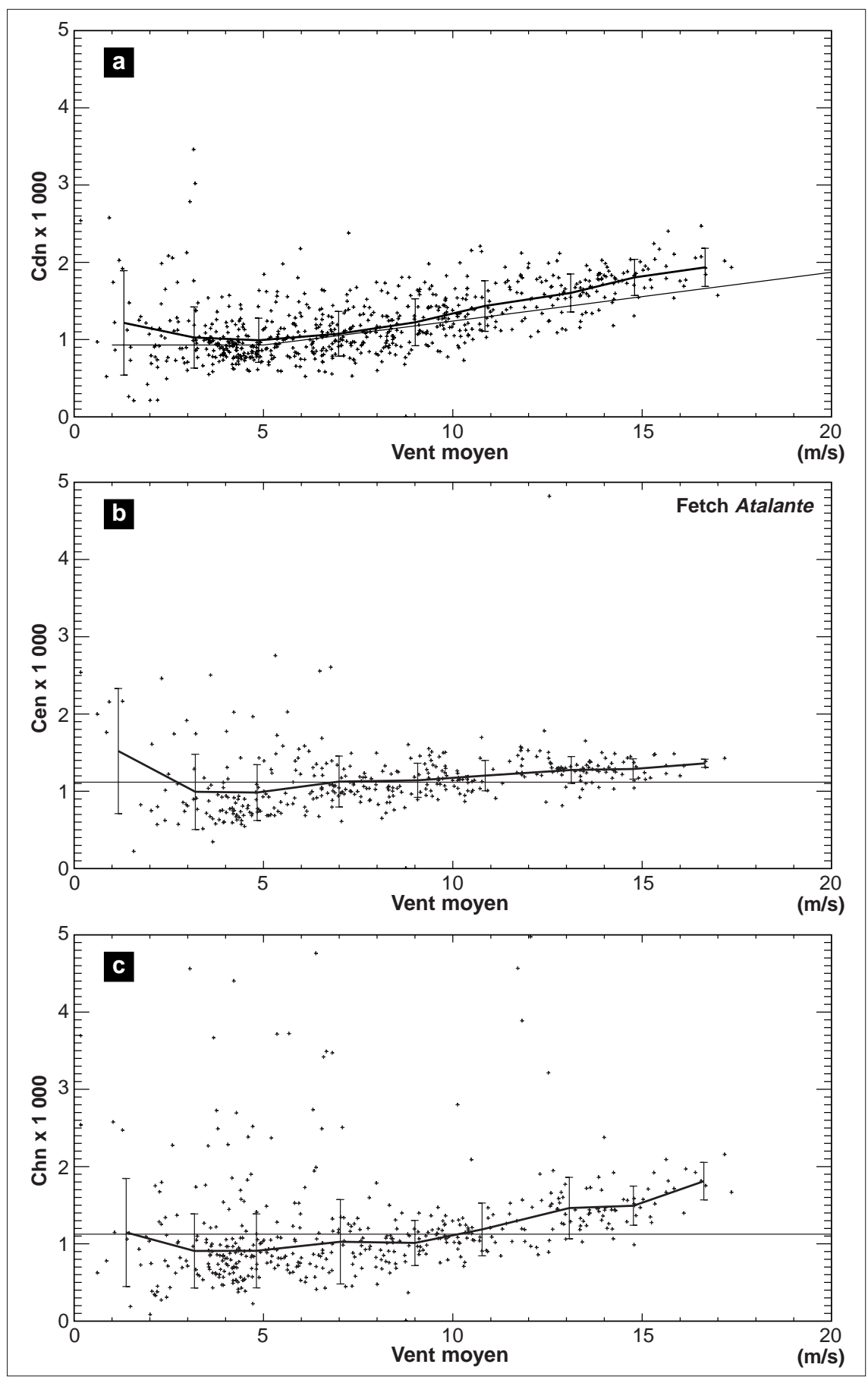

Figure 4 - Coefficients d'échange de quantité de mouvement (a) et de chaleur sensible (b) et latente (c) en fonction du vent moyen. Le vent et les coefficients sont ramenés à une altitude de $10 \mathrm{~m}$ au-dessus de la surface de la mer et à des conditions de stratification neutre. Le trait épais et les barres verticales associées représentent le coefficient moyen et son écart type, calculés dans des classes de vent de $2 \mathrm{~m} / \mathrm{s}$. Le trait plus fin correspond à la relation de $\mathrm{Smith}$ (1980) en (a) et de De Cosmo et al. (1996) en (b) et en (c). Analyse effectuée par H. Dupuis et C. Guérin.

Les deux quantités sont ramenées, suivant l'habitude pour ce type d'analyse, à des valeurs qui seraient observées à une altitude de $10 \mathrm{~m}$ au-dessus de la surface, en condition de stratification neutre. L'analyse préliminaire de ces résultats montre que la paramétrisation pour le flux de quantité de mouvement en cas de vent modéré (de 5 à $12 \mathrm{~m} . \mathrm{s}^{-1}$ ) est relativement proche de la relation de Smith (1980), fréquemment utilisée dans l'estimation des flux à l'interface océan-atmosphère, tandis qu'en cas de vent fort, les différences sont plus importantes. L'étude de l'influence respective des vagues et de la distorsion d'écoulement par le navire est en cours. En particulier, l'approche développée par le Centre 


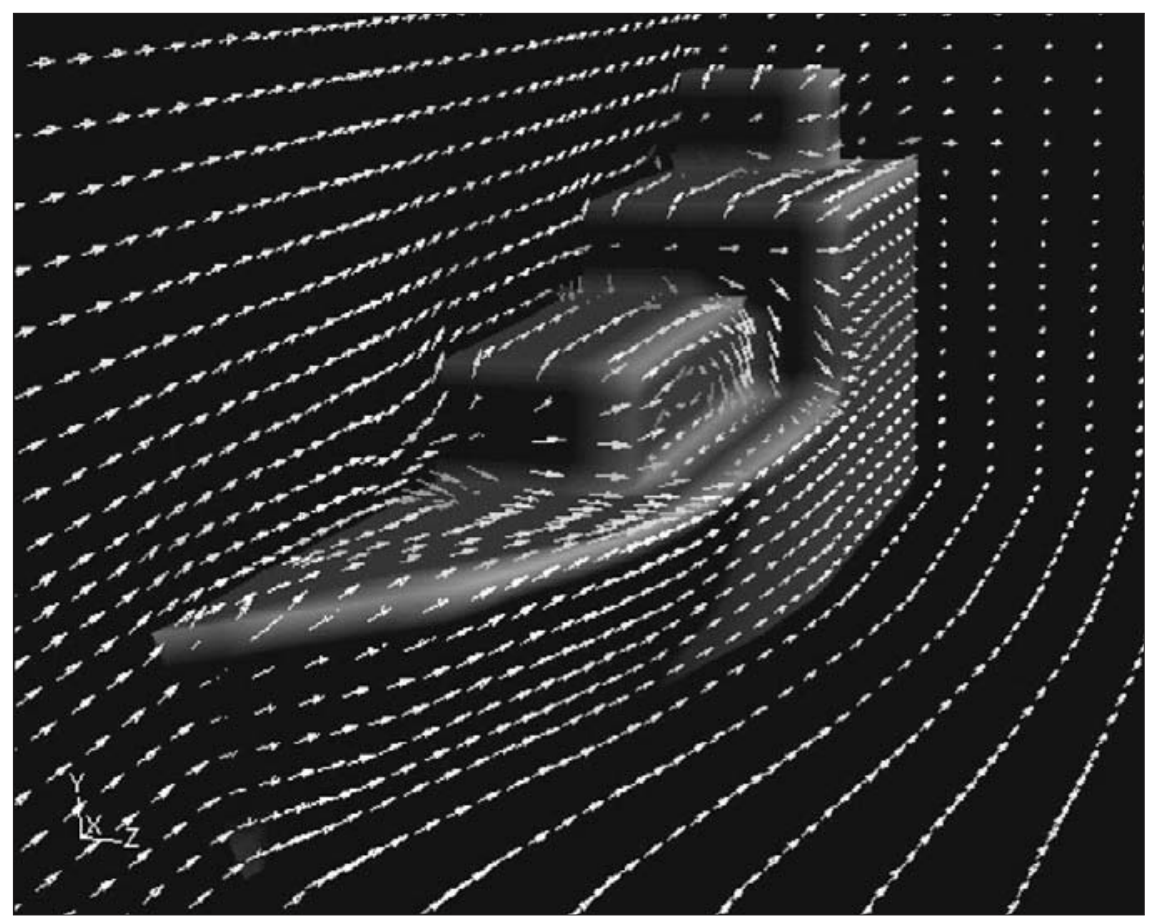

Figure 5 - Simulation de l'écoulement du vent autour de l'Atalante. Le navire est face à un vent de $10 \mathrm{~m} . \mathrm{s}^{-1}$. Les flèches montrent la direction du vent autour des superstructures. (Document extrait de Met Mar $\mathrm{n}^{\circ}$ 178)

d'aviation météorologique du CNRM pour estimer la distorsion d'écoulement autour des avions a été appliquée au cas du navire (figure 5 ; Nacass, 1999). Une première estimation de l'effet de cette distorsion a été obtenue dans une configuration particulière de vent, mais elle doit être complétée par des simulations d'écoulement dans les différentes configurations de vent et d'angles d'attaque du vent. Concernant l'influence des vagues, l'analyse sera couplée à une description de l'état de la mer combinant mesures in situ, mesures aéroportées et sorties des modèles de prévision des vagues, tel le modèle VAG de Météo-France.

En ce qui concerne les flux de chaleur, on observe une augmentation des coefficients d'échange avec le vent (figures $4 \mathrm{~b}$ et $4 \mathrm{c}$ ), ce qui apparaît comme un résultat nouveau par rapport aux observations de la campagne Hexos menée dans les années quatre-vingt en mer du Nord (De Cosmo et al., 1996).

\section{Mesures altimétriques du vent en situation de fetch limité}

Le modèle utilisé de façon opérationnelle pour restituer la vitesse du vent de surface, à partir du coefficient de rétrodiffusion mesuré par un radar altimétrique, a été établi de façon quasi empirique par comparaison avec des mesures de vent sur bouées. L'ensemble de ces mesures est représentatif de conditions océaniques moyennes, ce qui peut expliquer la dispersion importante observée lors des validations de ce modèle. Pour améliorer la précision de restitution de la vitesse du vent, des études récentes (Elfouhaily et al., 1998) prennent en compte la hauteur des vagues et un paramètre caractérisant l'âge des vagues. La campagne Fetch est l'occasion de poursuivre ces recherches, en particulier pour les états de mer jeune.

Durant la campagne, la zone Fetch a été échantillonnée six fois par l'altimètre de Topex et quatre fois par celui d'ERS 2. Quelques résultats préliminaires sont donnés ci-après, par vent de terre, pour les quatre premiers passages, trois de Topex et un d'ERS 2. Pour chaque passage, les mesures sont comparées aux sorties des modèles météorologiques Aladin, Arpège et CEPMMT, ainsi qu'aux mesures in situ disponibles.

Les champs de vent d'Arpège et la position des mesures par altimètre sont reportés sur la figure 6 . Les champs de vent correspondent à l'analyse disponible du modèle pour l'heure la plus proche de celle du passage du satellite. Le premier cas (a) correspond à un vent fort (mistral et tramontane), le 14 mars 1998, 

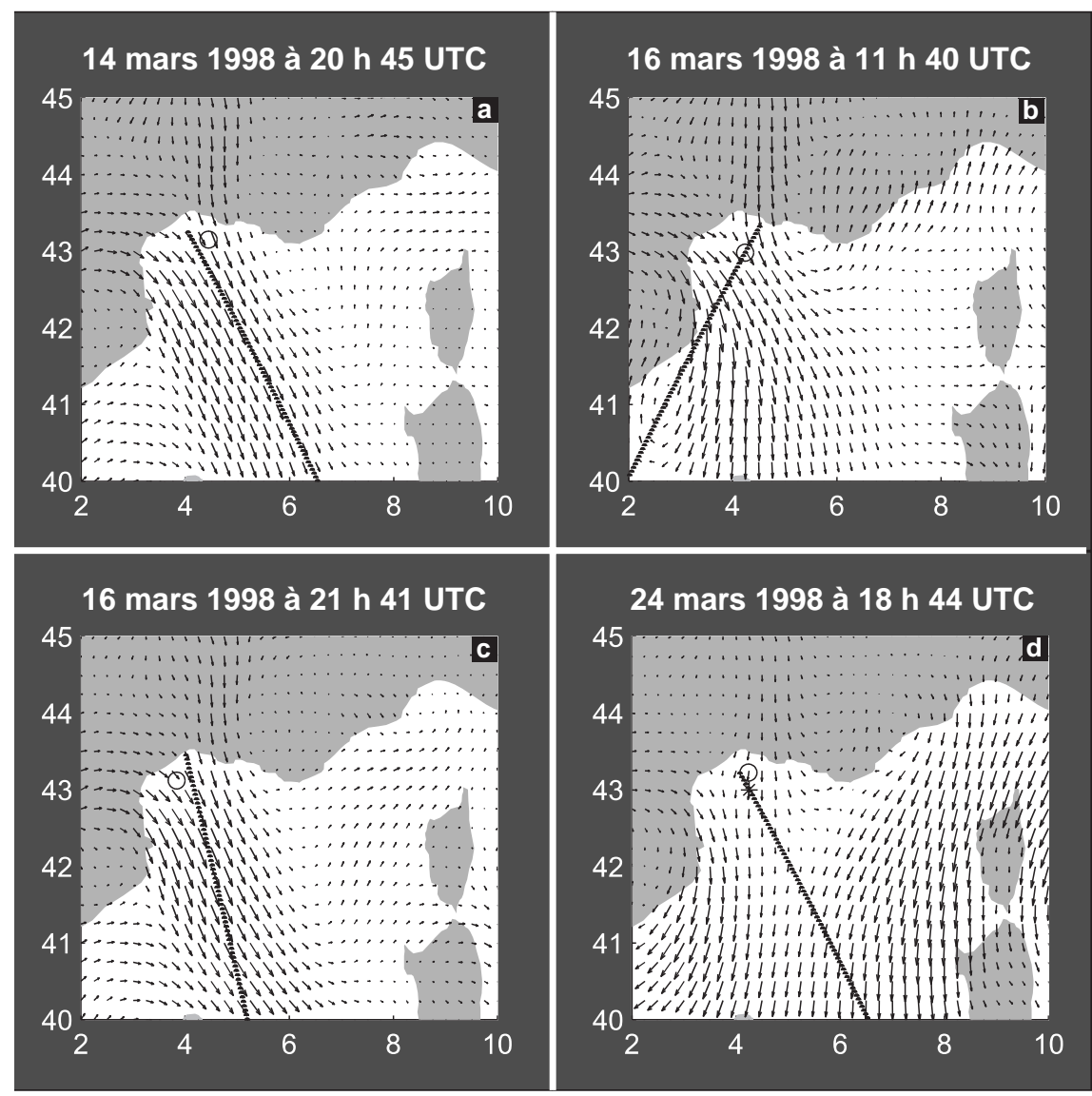

Figure 6 - Champs de vent analysés par le modèle Arpège disponibles à l'heure la plus proche de celle du passage du satellite altimétrique. (a) : Topex le 14 mars 1998 à $20 \mathrm{~h} 45$ UTC, analyse du 25 mars 1998 à 0 h UTC; (b) : Topex le 16 mars 1998 à 11 h 40 UTC, analyse du 16 mars 1998 à 12 h UTC ; (c) : ERS 2 le 16 mars 1998 à 21 h 41 UTC, analyse du 17 mars 1998 à 0 h UTC ; (d) : Topex le 24 mars 1998 à 18 h 44 UTC, analyse du 24 mars 1998 à 18 h UTC. La position de l'Atalante est marquée par un cercle, celle de la bouée Asis par une étoile et celle des mesures de l'altimètre par un trait gras.

en tout début de campagne. Les flux de nord et d'ouest convergent au-dessus du golfe du Lion. La vitesse de vent maximale estimée par le modèle est de $17 \mathrm{~m} . \mathrm{s}^{-1}$, autour du point situé à $42,5^{\circ}$ de latitude nord et $3,75^{\circ}$ de longitude est. La trace de Topex est globalement orientée dans la direction du vent. Le deuxième et le troisième cas (b et c), le 16 mars 1998, correspondent aussi à du mistral. La vitesse maximale du vent selon le modèle est de $13,2 \mathrm{~m} \cdot \mathrm{s}^{-1}$ (par $42,5^{\circ} \mathrm{N}$ et $3,5^{\circ} \mathrm{E}$ ) en (b) et de 15,9 $\mathrm{m} \cdot \mathrm{s}^{-1}$ en (c). En (b), le passage de Topex est presque perpendiculaire à la direction du vent. En (c), la mesure par altimètre est celle d'ERS 2. En (d), le 24 mars 1998, le vent souffle aussi du nord, mais la vitesse est plus faible que dans les cas précédents.

Les données satellitaires ont été comparées aux vents des modèles météorologiques, issus des analyses pour les modèles CEPMMT et Arpège, et des prévisions pour Aladin. Les vents des modèles ont été interpolés aux dates et aux positions des mesures altimétriques, tous les $5 \mathrm{~km}$ pour Topex et tous les $7 \mathrm{~km}$ pour ERS 2 (figure 7). Dans les trois cas, il s'agit de vent rapportés à une hauteur de $10 \mathrm{~m}$ audessus de la surface. Les mesures de vent effectuées sur l'Atalante (cercle) et sur la bouée Asis (étoile) sont également reportées sur la figure. Elles correspondent aux valeurs mesurées à l'altitude des capteurs (18 m et $7 \mathrm{~m}$, respectivement). Sur la figure 7a, on remarque qu'à proximité de la côte, la vitesse du vent déduite de l'altimètre de Topex est inférieure aux données des modèles, mais qu'elle atteint un maximum de $16,6 \mathrm{~m} . \mathrm{s}^{-1}$ bien plus élevé que celui des modèles. À proximité de la côte, des différences importantes existent entre les modèles, en particulier entre Arpège et celui du CEPMMT. Les résolutions spatiales des trois modèles sont différentes (maille carrée en latitude et longitude de $0,5^{\circ}$ pour le CEPMMT, $0,25^{\circ}$ pour Arpège et $0,1^{\circ}$ pour Aladin) et les valeurs extrêmes peuvent, de ce fait, être lissées différemment. La mesure de l'Atalante, moyennée sur 10 minutes, est de 


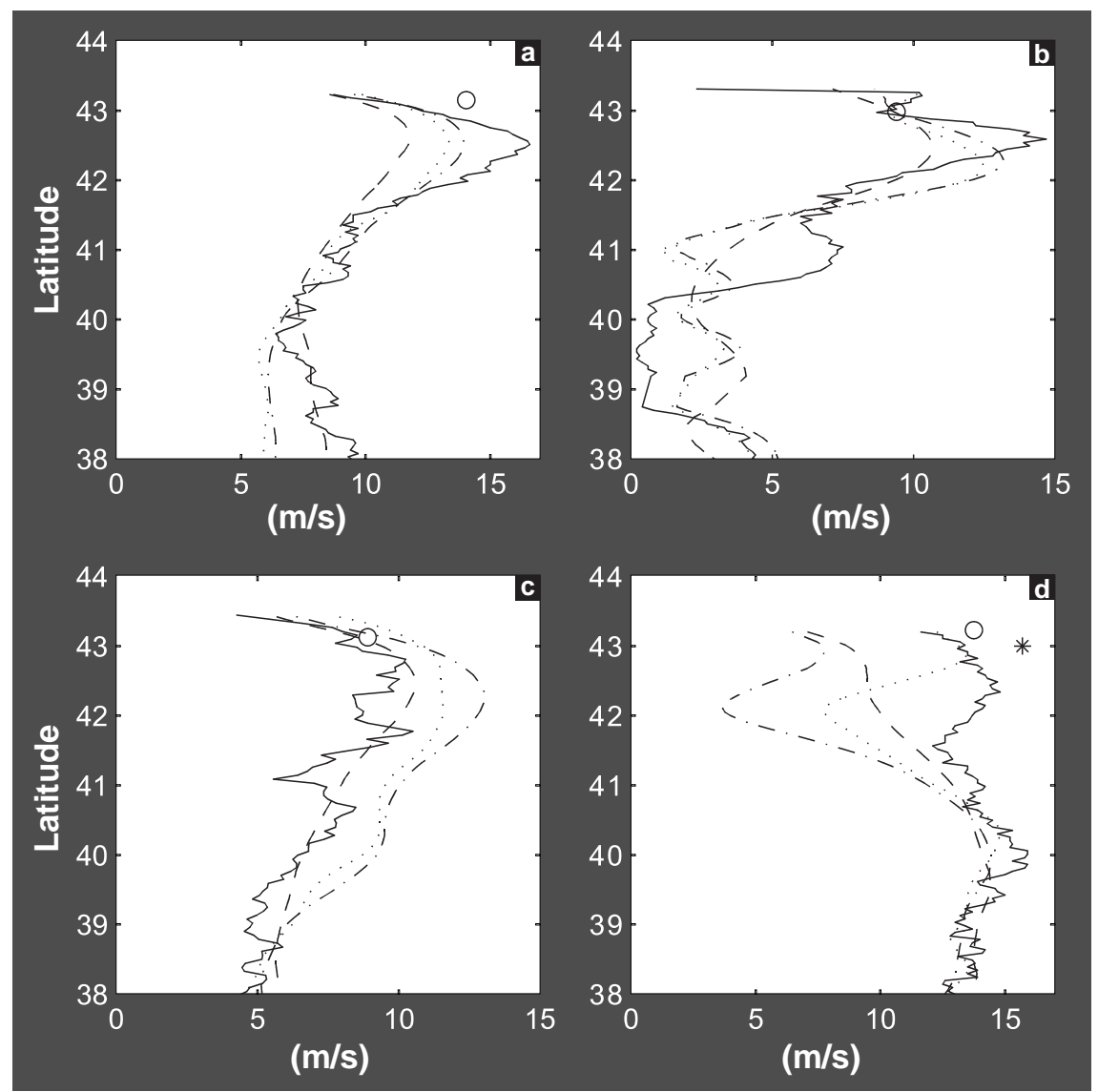

Figure 7 - Comparaison, le long de la trace de l'altimètre, de la vitesse du vent déduite de l'altimètre (trait plein) et des vitesses du vent obtenues par interpolation des sorties des modèles Aladin (pointillé), Arpège (tiret et pointillé) et CEPMMT (tiret). Mesures de l'Atalante (cercle) et de la bouée Asis (étoile). Les figures $a, b, c, d$ correspondent aux mêmes dates et aux mêmes heures que celles de la figure 6 . Analyse effectuée par P. Queffeulou.

l'ordre de $14 \mathrm{~m} \cdot \mathrm{s}^{-1}$ alors que la mesure correspondante de Topex est de $11 \mathrm{~m} \cdot \mathrm{s}^{-1}$. Même après correction des observations de l'Atalante pour estimer le vent à la hauteur standard de $10 \mathrm{~m}$, une différence significative existe puisque le vent de l'Atalante est alors de $13,2 \mathrm{~m} \cdot \mathrm{s}^{-1}$. La forte augmentation de la vitesse du vent observée en allant de la côte vers le large résulte probablement du changement de rugosité de surface lors de la transition terre-mer, ainsi que de la diminution vers le large de la stratification de la couche de surface, par réchauffement des couches d'air au voisinage de la surface de la mer, plus chaude.

Sur la figure 7b, on constate qu'au voisinage de la position de l'Atalante, les données du bateau, des modèles et de l'altimètre sont assez cohérentes. La trace de l'altimètre traverse perpendiculairement un flux de nord-ouest et met en évidence un étroit couloir de vent fort, en général atténué par les modèles du fait de leur résolution spatiale. Sur la figure $7 \mathrm{c}$, le comportement relatif des modèles et de l'altimètre est inverse de celui observé en 7a, bien que les conditions météorologiques se ressemblent. Pour le cas de la figure $7 \mathrm{~d}$, les modèles fournissent des vitesses très différentes. Les vitesses mesurées sur l'Atalante et sur la bouée Asis sont plus proches des mesures de l'altimètre que des données des modèles, et cela reste vrai si l'on rapporte les mesures in situ à la hauteur standard de $10 \mathrm{~m}$.

Cette première analyse montre que, dans ces quelques situations de vent de terre, les données issues de l'altimètre, des modèles et des capteurs in situ peuvent être très différentes à proximité de la côte. Dans ces conditions, l'utilisation des modèles pour estimer l'impact du fetch sur la mesure de l'altimètre ne semble pas être une solution satisfaisante. La cohérence des mesures altimétriques du vent et de la hauteur des vagues sera testée à l'aide des relations classiques existant entre vent, fetch et hauteur des vagues. Les mesures aéroportées, en particulier pour le cas du 24 mars 1998 où de larges différences sont observées, pourront contribuer à ce travail. Quand les mesures in situ de vitesse 


\section{Observations par lidar dans un cas de mistral}

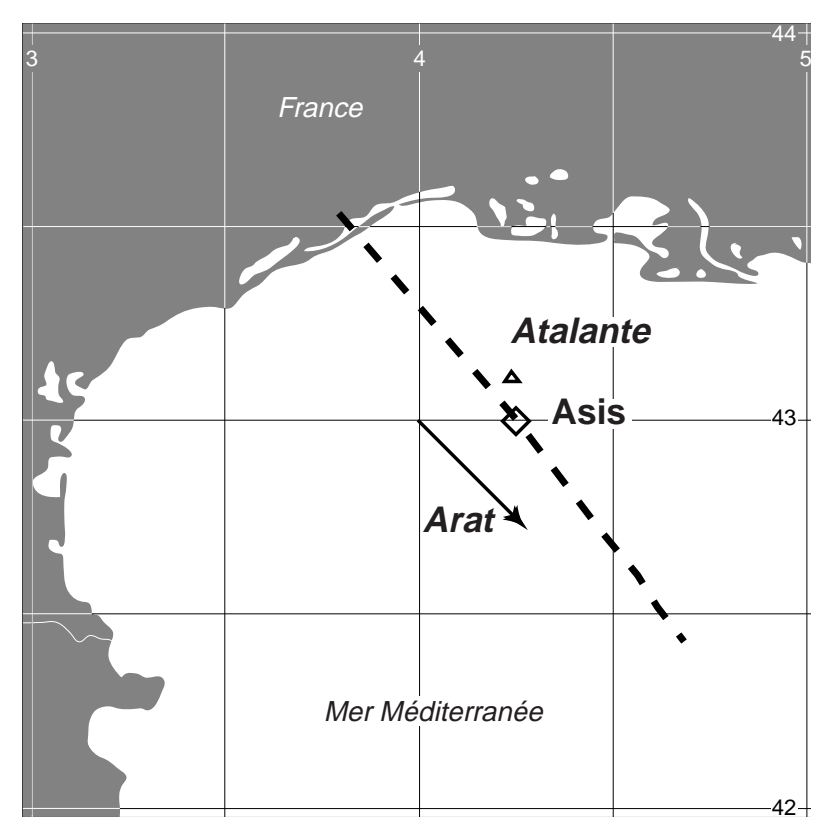

Figure 8 - Trace au sol des mesures lidar du 24 mars 1998 (ligne en tirets, sens de déplacement de l'avion indiqué par la flèche) et posi-

tion des mesures in situ du navire Atalante et de la bouée Asis. de vent et de vitesse de frottement auront été validées, elles seront utilisées pour tester les modèles altimétriques bifréquence associant hauteur des vagues et coefficients de rétrodiffusion.

Le lidar aéroporté Leandre 2 (Bruneau et al., en préparation) permet d'obtenir en air clair deux paramètres critiques pour l'étude des flux turbulents de chaleur à la surface de la mer : l'humidité spécifique et le vent de surface (voir encadré ci-après). Au-dessus de la Méditerranée, il a également permis d'analyser l'évolution à méso-échelle du vent de surface avec une résolution horizontale de l'ordre de $80 \mathrm{~m}$. Nous présentons ici quelques résultats préliminaires obtenus lors de l'épisode de mistral du 24 mars 1998. La figure 8 montre la zone expérimentale ainsi que la trace au sol d'une partie du plan de vol de l'Arat, embarquant Leandre 2, effectué le 24 mars 1998 entre 16 h 20 et 16 h 55 UTC.

\section{Le lidar Leandre 2}

Le lidar aéroporté Leandre 2 permet de mesurer le profil de I'humidité spécifique, par absorption différentielle. La réflectivité lidar, due aux particules et aux molécules atmosphériques, est mesurée quasi simultanément à deux longueurs d'onde très proches. Une de ces longueurs d'onde est située sur une raie d'absorption de la vapeur d'eau, l'autre est prise comme référence. La différence de transmission entre les signaux mesurés aux deux longueurs d'onde permet de déterminer le contenu total et le profil vertical du rapport de mélange de la vapeur d'eau. Dans les conditions de l'expérience Fetch, le domaine d'étude concerne les 3,5 premiers kilomètres de l'atmosphère et la résolution horizontale et verticale est d'environ $300 \mathrm{~m}$. Le lidar permet également de fournir des informations sur les échanges à la surface de la mer. La longueur d'onde de mesure étant très courte devant celle des ondes de surface, le signal lidar diffusé par la surface peut être relié de façon théorique à la variance des inclinaisons des miroirs élémentaires dues aux ondes de capillarité-gravité dont le taux de croissance dépend directement du flux de quantité de mouvement (ou du vent). Au-dessus de la Méditerranée, le lidar a ainsi permis, pendant Fetch, d'analyser l'évolution à méso-échelle du vent de surface avec une résolution horizontale de l'ordre de $80 \mathrm{~m}$.

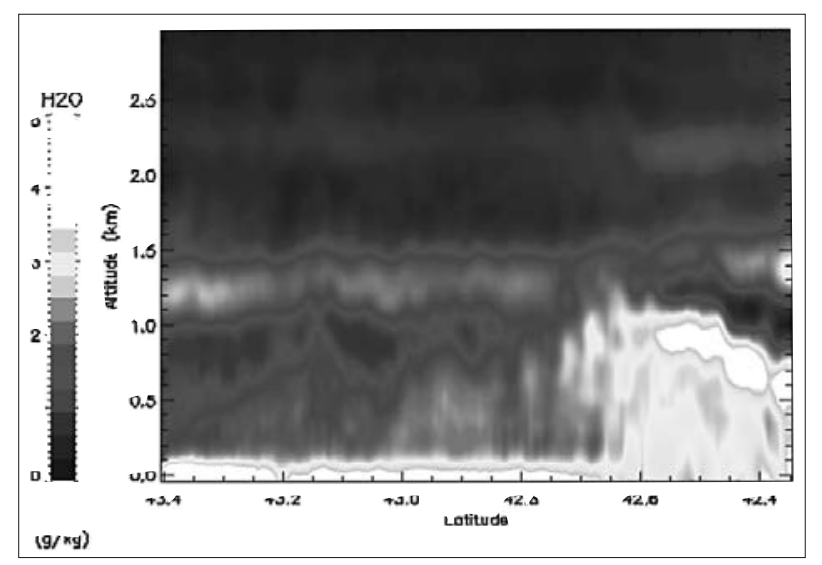

Figure 9 - Coupe verticale du rapport de mélange en vapeur d'eau, déduit des mesures de Leandre 2 le long de l'axe de vol indiqué sur la figure 8 . Analyse effectuée par C. Flamant et J. Pelon.
La figure 9 présente la coupe verticale du champ de vapeur d'eau déduit des mesures de Leandre 2 le long de l'axe de vol tracé sur la figure 8. Dans cet exemple, les observations montrent le développement d'une couche limite atmosphérique interne (CLAI) que l'on reconnaît sur la figure 9 par le fait que le gradient vertical d'humidité caractérisant la zone d'interface entre la couche bien mélangée et l'atmosphère libre s'élève en altitude au fur et à mesure que l'on s'éloigne de la côte pour des latitudes comprises entre $43,4^{\circ} \mathrm{N}$ et $42,6^{\circ} \mathrm{N}$ environ. Au sein de cette CLAI, l'humidité observée est plus importante que celle de l'air advecté depuis le continent et que l'on retrouve au large, à des latitudes inférieures à $42,6^{\circ} \mathrm{N}$. Le mécanisme conduisant à la génération de la CLAI est l'intensification des flux turbulents de chaleur à la surface, due à l'accélération de l'écoulement au passage terre-mer et à l'augmentation du contraste thermique entre l'air et la mer. Au large, la CLAI atteint un état d'équilibre et son épaisseur décroît quand on s'éloigne encore, en raison de la subsidence à l'échelle synoptique. Les valeurs d'humidité spécifique déduites des mesures de Leandre 2 dans la CLAI sont en bon accord avec celles mesurées in situ. 


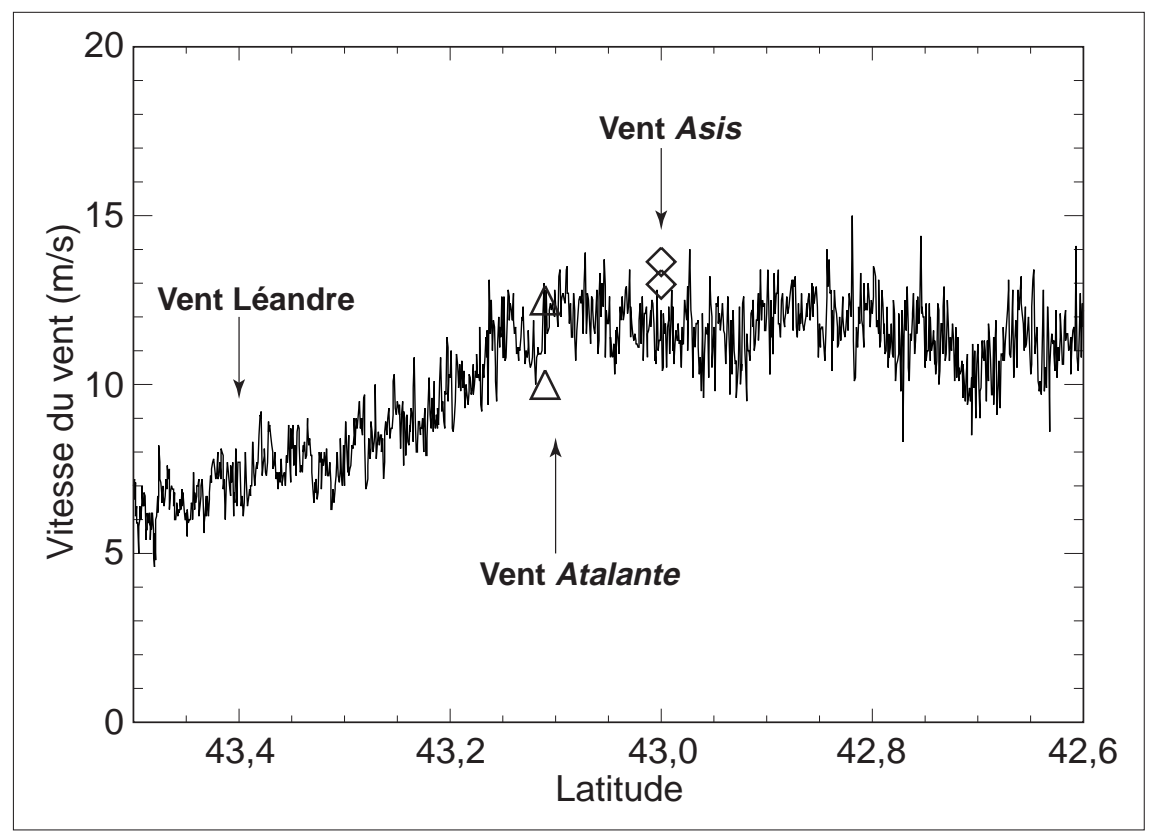

Figure 10 - Vent de surface à $10 \mathrm{~m}$ estimé à partir des mesures de Leandre 2 sur l'axe de vol indiqué à la figure 8. Analyse effectuée par C. Flamant et J. Pelon.

La figure 10 montre les variations du vent de surface, à $10 \mathrm{~m}$ au-dessus du sol, estimé à partir des mesures de Leandre 2, et les mesures de vent in situ sur le même axe de vol. Le vent estimé à partir de Leandre 2 a été obtenu en utilisant un modèle empirique (Flamant et al., 1998 ; Cox et Munk, 1954). On observe l'accélération de l'écoulement liée à la diminution de la rugosité au passage terre-mer, entre 43,5 et $43,1^{\circ} \mathrm{N}$, puis un vent relativement constant entre 43,1 et $42,6^{\circ} \mathrm{N}$. Une première comparaison a été effectuée avec les mesures de vent faites à bord de l'Atalante, à une hauteur de $18 \mathrm{~m}$, et sur la bouée Asis, à une hauteur de $7 \mathrm{~m}$, aux heures de passage du lidar. Elle a donné des résultats satisfaisants, d'autant plus que les mesures de vent in situ ne sont pas rapportées à la hauteur standard de $10 \mathrm{~m}$. Il est évident qu'un plus grand nombre de comparaisons est nécessaire afin de valider cette approche. Cependant, cette première analyse montre que le lidar est capable de fournir une information sur la variation spatiale du vent de surface, qui pourrait être utilisée en complément des mesures de profil d'humidité pour estimer le flux de chaleur latente.

Évolution des vagues dans une situation de mistral

Le 24 mars 1998 a été choisi comme l'un des cas à étudier en priorité, car il correspond à une situation de mistral. Parmi les résultats préliminaires obtenus pour ce cas, on illustre ici ceux qui concernent la variation du spectre des vagues en fonction de la distance à la côte.

La figure 11 montre la densité spectrale des hauteurs de vagues en fonction de leur fréquence, obtenue à partir du radar aéroporté Ressac en différentes positions de mesure correspondant à différentes distances de fetch. On retrouve le comportement attendu pour l'évolution des vagues à fetch court : la fréquence des vagues dominantes diminue avec le fetch, l'énergie maximale et l'énergie totale du spectre augmentent. De façon plus quantitative, on trouve que l'évolution de la fréquence du maximum d'énergie en fonction du fetch est en accord avec la relation empirique obtenue au cours de l'expérience Jonswap (Hasselmann et al., 1993), pour une vitesse de vent du même ordre (10 à $\left.15 \mathrm{~m} \cdot \mathrm{s}^{-1}\right)$. Par contre, pour cette même vitesse du vent, l'augmentation de la hauteur significative des vagues, qui est reliée à la variance totale du spectre des vagues, semble plus rapide que celle qui est donnée par les résultats de Jonswap. L'analyse d'autres cas est nécessaire pour conclure sur le caractère systématique de ce résultat.

Une analyse des distributions angulaires du spectre des vagues a été aussi entreprise sur ce cas. Les paramètres caractérisant l'étalement angulaire, calculés à partir des données de Ressac, sont en bon accord avec ceux donnés par la 
Figure 11 - Spectres non directionnels des vagues obtenus à partir des mesures du radar aéroporté Ressac. Chaque courbe correspond à une gamme de distance de fetch. Les traits continus sont relatifs aux données acquises lors du trajet aller de l'avion (de 17 h 47 à 17 h 58 UTC), les traits pointillés aux données acquises lors du trajet retour de l'avion (de 18 h 14 à 18 h 53 UTC). Analyse effectuée par D. Hauser et C. Quentin.

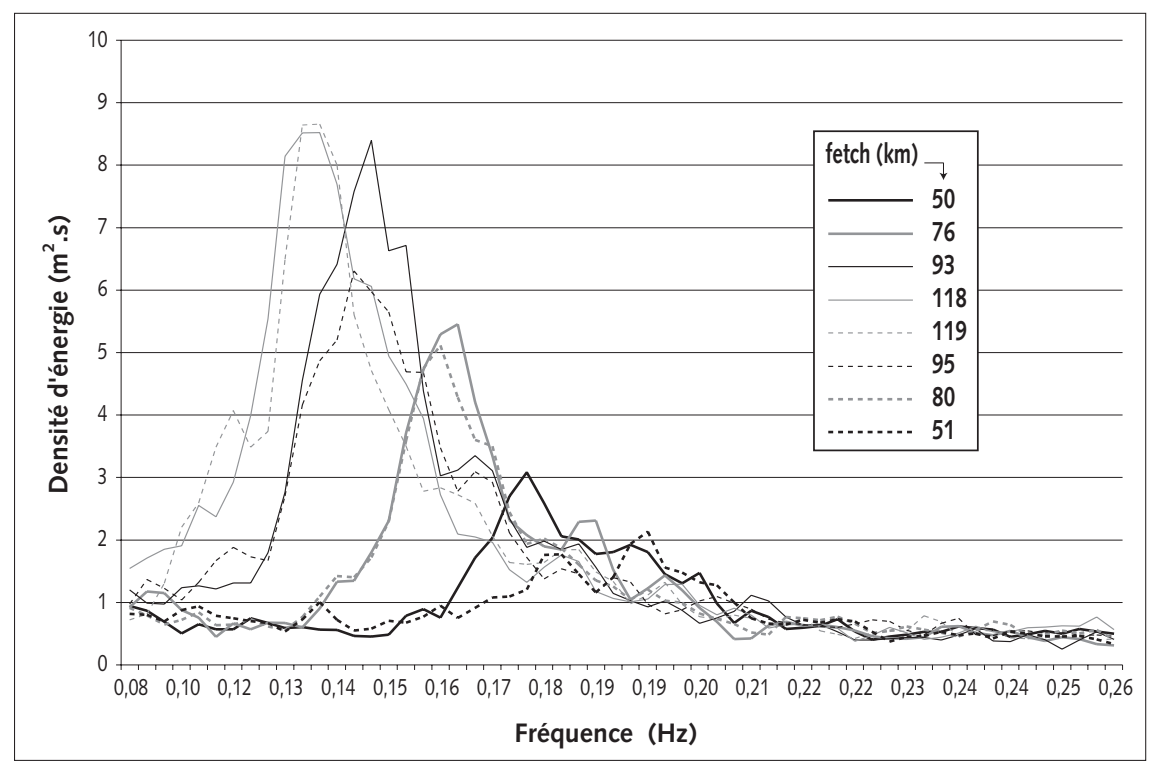

bouée FIMR au point B. L'analyse de l'ensemble des données de Ressac montre que l'étalement angulaire est minimal pour des fréquences de vagues égales à 1,2 fois la fréquence du pic du spectre et qu'il ne semble pas dépendre du fetch. Ce résultat, s'il est confirmé par l'analyse des autres cas, est important pour la modélisation numérique des états de la mer.

Pour généraliser cette analyse, les résultats doivent être confrontés à d'autres observations. Il est important de pouvoir en tirer des conclusions sur la dépendance des caractéristiques du spectre en fonction du vent et du fetch, notamment pour améliorer la modélisation des états de la mer et pour évaluer l'influence des vagues sur les flux turbulents à l'interface océan-atmosphère.

\section{Le radar Ressac}

Le radar Ressac permet de mesurer le vent à la surface de l'océan et la hauteur des vagues. Il fonctionne à la fréquence de $5,35 \mathrm{GHz}$, en polarisation horizontale. Dans son mode standard d'utilisation, il vise la surface avec une incidence voisine de $14^{\circ}$ par rapport à la verticale et un faisceau balayant la surface sur

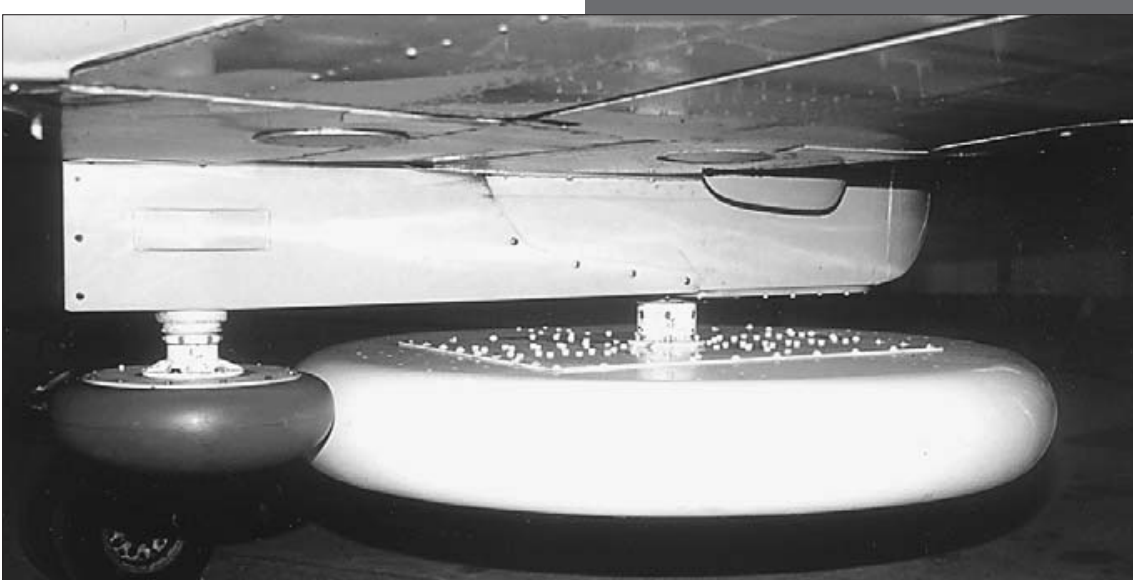
$360^{\circ}$; ces données sont ensuite traitées pour restituer le spectre des hauteurs de vagues. On fournit généralement un spectre directionnel tous les $10 \mathrm{~km}$ le long de la trace au sol de l'avion, avec une résolution de $15^{\circ}$ pour la direction de propagation des vagues. La gamme de longueur d'onde observable à partir de Ressac est d'environ 40 à $350 \mathrm{~m}$, avec une résolution d'environ $10 \%$ de la longueur d'onde observée. Un mode complémentaire a été utilisé pendant Fetch pour estimer le vent. Dans ce cas, l'antenne est gardée en position fixe sur le côté de l'avion, tandis que ce dernier effectue des vols en cercle.

Vue extérieure du Merlin IV montrant. fixées sous le fuselage, les deux antennes du radar aéroporté Ressac. La grosse antenne, claire, est l'antenne de réception; la petite antenne, sombre, l'antenne d'émission. (Photo Météo-France, P. Taburet) ce qui permet de viser avec une incidence moyenne de $34^{\circ}$ par rapport à la verticale. L'analyse de ces observations permet de restituer la vitesse et la direction du vent (pour la vitesse, un modèle empirique est utilisé, reliant section efficace radar et vent). 
CONCLUSION

a campagne Fetch a permis de recueillir des mesures de qualité dans des conditions peu étudiées (vent fort, mer en développement, zone côtière) en combinant des types d'observations complémentaires dans l'atmosphère, dans l'océan et à sa surface ainsi que par télédétection. L'analyse des données de la campagne Fetch a bien commencé, mais elle est encore en pleine phase de développement. Dans les mois et les années qui viennent, on attend des résultats dans plusieurs domaines.

Les résultats sur la paramétrisation des flux turbulents préciseront la dépendance de ces flux par rapport aux conditions moyennes, en particulier pour le vent, et les effets éventuels de l'état de la mer. Sur le plan méthodologique, les compétences développées pour la mesure et l'analyse des flux turbulents ont été utilisées dans d'autres expériences, comme l'expérience Equalant en 1999, ou seront mises à profit prochainement (expérience Pomme en 2000-2001, etc.). C'est grâce à l'ensemble de ces campagnes que la communauté scientifique pourra mieux comprendre et quantifier le rôle des échanges turbulents et radiatifs dans la dynamique des premières couches océaniques et atmosphériques.

Les études sur les vagues menées dans le cadre de Fetch devraient permettre de faire progresser la prévision des états de la mer, notamment dans les conditions côtières en Méditerranée. On sait que la mer levée par des coups de vent forts peut conduire à des situations dramatiques, souvent mal prévues à cause des limites actuelles des modèles d'état de la mer. L'amélioration de ces modèles nécessite une meilleure qualité des valeurs du vent utilisés pour le forçage, une meilleure représentation de la génération et de l'évolution des vagues et enfin le développement de méthodes d'assimilation de données. Sur tous ces points, on attend des avancées significatives grâce à l'analyse des données de Fetch.

En télédétection hyperfréquence par les radars et les radiomètres, la détermination des paramètres géophysiques par inversion du signal télédétecté repose la plupart du temps sur des modèles empiriques ou semi-empiriques qui présentent des limites importantes dans leur application à des cas particuliers, tels que les zones côtières. Grâce à la combinaison des observations locales et des mesures par télédétection, la base de données de l'expérience Fetch constitue une opportunité nouvelle pour améliorer la compréhension et l'inversion des relations entre signal de télédétection et paramètres géophysiques. Il en est de même pour le domaine plus novateur de la mesure des propriétés du vent de surface par lidar.

Concernant la circulation océanique dans le golfe du Lion, on attend beaucoup de la combinaison entre observation et modélisation pour mieux comprendre l'interaction entre les différents processus : circulation induite par les coups de vent et les modifications de flux de chaleur, circulation générale du bassin représentée par le courant liguro-provençal, apports d'eau douce du Rhône. L'influence des vagues sur le frottement de fond devrait également être mieux représentée. Grâce à ces développements, l'étude des écosystèmes du golfe du Lion pourra progresser en reposant sur une modélisation physique des transports de matière (nutriments, sédiments, etc.).

Par la diversité des sujets abordés et des moyens utilisés, l'expérience Fetch a su rassembler des équipes de différents organismes, travaillant dans des domaines différents mais complémentaires. Les collaborations mises en place avant, pendant et après l'expérience permettent de progresser rapidement sur les objectifs annoncés.

\section{BIBLIOGRAPHIE}

Bruneau D., P. Quaglia, C. Flamant, M. Meissonnier et J. Pelon, en préparation : The airborne lidar Leandre 2 for water vapor profiling in the troposphere; description and first results. Appl. Opt.

Chalon J.-P., M. André, J.-L. Brenguier, A. Druilhet, J.-F. Gayet, P. Flamant, B. Guillemet, D. Hauser, Y. Kerr, J. Pelon, M. Ravaut et D. Tanré, 1998 : Les avions français de recherche atmosphérique et de télédétection : bilan et perspectives. $L a$ Météorologie $8^{\mathrm{e}}$ série, 22, 14-44.

Cox C. et W. Munk, 1954 : Measurements of roughness of the sea surface from photographs of the sun's glitter. J. Opt. Soc. of America, 44, 11, 832-850.

De Cosmo J., K. B. Katsaros, S. D. Smith, R. J. Anderson, W. A. Oost, K. Bumke et H. Chadwick, 1996 : Air sea exchange of sensible heat and water vapor: the Hexos results. $J$. Geophys. Res., 101, 12001-12016. 
Elfouhaily T., J. Gourrion, B. Chapron et D. Vandemark, 1998 : Estimation of wind stress using dual-frequency Topex data. J. Geophys. Res., 103, C11, 25101-25108.

Eymard L., S. Planton, P. Durand, C. Le Visage, P.-Y. Le Traon, L. Prieur, A. Weill, D. Hauser, J. Rolland, J. Pelon, F. Baudin, B. Benech, J.-L. Brenguier, G. Caniaux, P. De Mey, E. Dombrowski, A. Druilhet, H. Dupuis, B. Ferret, C. Flament, P. Flamant, F. Hernandez, D. Jourdan, K. Katsaros, D. Lambert, J.-M. Lefèvre, P. Le Borgne, B. Le Squere, A. Marsoin, H. Roquet, J. Tournadre, V. Trouillet, A. Tychensky et B. Zakardjian, 1996 : Study of the air-sea interactions at the mesoscale; the Semaphore experiment. Ann. Geophys., 14, 986-1015.

Eymard L., G. Caniaux, H. Dupuis, L. Prieur, H. Giordani, R. Troadec, D. Bourras, C. Guérin, P. Le Borgne, A. Brisson et A. Marsoin, 2000 : Surface fluxes in the North Atlantic current during the Catch-Fastex experiment. Quart. J. Roy. Meteor Soc., sous presse.

Flamant C., V. Trouillet, P. Chazette et J. Pelon, 1998 : Wind speed dependence of atmospheric boundary layer optical properties and ocean surface reflectance as observed by airborne backscatter lidar. J. Geophys. Res., 103, C11, 25137-25158.

Geernaert G. L, 1990 : Bulk parameterizations for the wind stress and heat fluxes. In Surface waves and Fluxes, vol. 1. Sous la direction de Geernaert et Plant. Kluwer Academic Publisher, $336 \mathrm{p}$.

Graber H. C., E. A. Terray, M. A. Donelan, W. M. Drennan, J. C. Van Leer et D. B. Peters, 2000 : Asis, a new air-sea interaction spar buoy; design and performance at sea. $J$. Atmos. Ocean. Technol., sous presse.

Hasselmann K. et al., 1973 : Measurements of wind-wave growth and swell decay during the joint North Sea wave project (Jonswap). Ergänzungsheft zur Deutschen Hydrographischen zeitschrift, 1-95.

Hauser D., G. Caudal, G. J. Rijckenberg, D. Vidal-Madjar, G. Laurent et P. Lancelin, 1992 : Ressac, a new airborne FM/CW radar ocean wave spectrometer. IEEE Trans. Geosci. Remote Sens., 30, 5, 981-995.

Lachaud G., 1998 : Un mât sur l'Atalante. Met Mar, 181, 4-7.

Nacass P., 1999 : Utilisation de la modélisation des écoulements aérodynamiques pour la mesure atmosphérique à bord du navire océanique l'Atalante. Météo-France, Note de centre $\mathrm{n}^{\circ} 22$ du CNRM, Toulouse.

Smith S. D., 1980 : Wind stress and heat flux over the ocean in gale force winds. J. Phys. Oceanogr., 10, 709-726. 